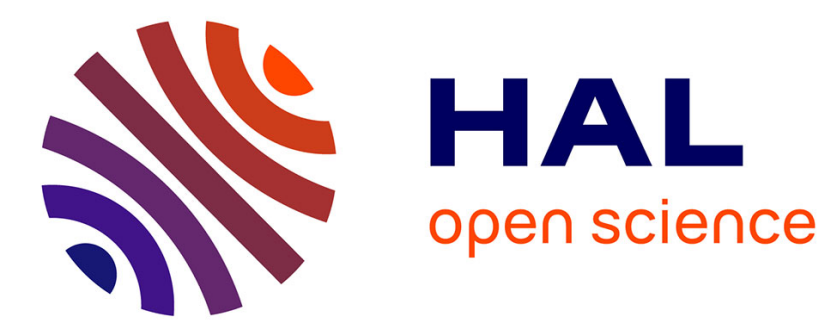

\title{
Limit laws for random matrix products
}

\author{
Jordan Emme, Pascal Hubert
}

\section{To cite this version:}

Jordan Emme, Pascal Hubert. Limit laws for random matrix products. Mathematical Research Letters, 2018, 25 (4), pp.1205 - 1212. 10.4310/MRL.2018.v25.n4.a7 . hal-01623840v2

\section{HAL Id: hal-01623840 \\ https://hal.science/hal-01623840v2}

Submitted on 8 Dec 2017

HAL is a multi-disciplinary open access archive for the deposit and dissemination of scientific research documents, whether they are published or not. The documents may come from teaching and research institutions in France or abroad, or from public or private research centers.
L'archive ouverte pluridisciplinaire HAL, est destinée au dépôt et à la diffusion de documents scientifiques de niveau recherche, publiés ou non, émanant des établissements d'enseignement et de recherche français ou étrangers, des laboratoires publics ou privés. 


\title{
Limit laws for random matrix products
}

\author{
Jordan Emme* and Pascal Hubert ${ }^{\dagger}$
}

\begin{abstract}
In this short note, we study the behaviour of a product of matrices with a simultaneous renormalization. Namely, for any sequence $\left(A_{n}\right)_{n \in \mathbb{N}}$ of $d \times d$ complex matrices whose mean $A$ exists and whose norms' means are bounded, the product $\left(I_{d}+\frac{1}{n} A_{0}\right) \ldots\left(I_{d}+\frac{1}{n} A_{n-1}\right)$ converges towards $\exp A$. We give a dynamical version of this result as well as an illustration with an example of "random walk" on horocycles of the hyperbolic disc.
\end{abstract}

\section{Introduction}

Products of random matrices - or cocycles - are generally studied and well understood via ergodic theory, martingales on Markov chains, or spectral theory for instance. For some literature in this direction, one can look at a book such as [1], the surveys [3,4]. Indeed, results like the Osseledec theorem give a precise asymptotic behaviour of a product of random matrices. It provides informations like the logarithmic growth rate of the norm of the matrices. However, in [2] we encountered a random product of matrices that did not fit the usually studied case. Indeed, understanding the limit of the characteristic functions of a renormalized sequence of probability measures had to be achieved by understanding, for any parameter $t$, a random product of matrices of the form $\left(A_{X_{0}}+\frac{t}{n} B_{X_{0}}\right) \ldots\left(A_{X_{n}}+\frac{t}{n} B_{X_{n}}\right)$ where $\left(X_{n}\right)_{n \in \mathbb{N}}$ is a binary sequences and $A_{0}, A_{1}, B_{0}, B_{1}$ are fixed $2 \times 2$ matrices. The scale of normalization is different from the standard one, thus the result is more precise. We obtained a convergence of the matrices instead of convergence of the logarithm of the norms. Nevertheless, the method involved heavily relied on the properties of the matrices and, as such, was ad hoc for the problem we were interested in. However, in a an effort to replicate and generalize this type of random product of matrices (namely by understanding Corollary 3.2), we stumbled upon a surprising general property of these types of products that we explicit in Theorem 1.1.

Theorem 1.1. Let $\left(A_{n}\right)_{n \in \mathbb{N}}$ be a sequence of $d \times d$ complex matrices satisfying

$$
\lim _{n \rightarrow+\infty} \frac{1}{n} \sum_{k=0}^{n-1} A_{k}=A
$$

and such that $\left(\frac{1}{n} \sum_{k=0}^{n}\left\|A_{k}\right\|\right)_{n \in \mathbb{N}^{*}}$ is bounded for a norm $\|\cdot\|$ by $\alpha$.

Define, for any $t$ in $\mathbb{C}$ and any positive integer $n$

$$
\Pi_{n}(t)=\left(I_{d}+\frac{t}{n} A_{0}\right) \ldots\left(I_{d}+\frac{t}{n} A_{n}\right) .
$$

\footnotetext{
*Université Paris-Sud, CNRS, LMO, UMR 8628, 91405 Orsay, France. E-mail: jordan.emme@math.u-psud.fr

†Aix-Marseille Université, CNRS, Centrale Marseille, I2M, UMR 7373, 13453 Marseille, France. E-mail: pascal.hubert@univ-amu.fr
} 
Then,

$$
\forall t \in \mathbb{C}, \lim _{n \rightarrow+\infty} \Pi_{n}(t)=\exp (t A) .
$$

Remark 1.2. Here is an heuristic explanation of the statement of Theorem 1.1. Consider the problem at the level of the Lie algebra. The main term is $\frac{t}{n} \sum_{k=0}^{n-1} A_{k}$ and its limit $t A$. The limit of $\Pi_{n}$ is the exponential of the limit in the Lie algebra. In a sense, at this scale, the behavior of the product is directed by the behavior of the sum $\sum_{k=0}^{n-1} A_{k}$ in the Lie algebra.

An elementary version of Theorem 1.1 for complex numbers is the following classical

Lemma 1.3. Let $\left(u_{n}\right)_{n \in \mathbb{N}}$ be a bounded complex sequence whose mean converges towards $l$. Then

$$
\lim _{n \rightarrow+\infty} \prod_{k=0}^{n-1}\left(1+\frac{u_{k}}{n}\right)=e^{l} .
$$

Acknowledgement We wish to thank Jayadev Athreya and Samuel Lelièvre for their interest in this problem and for sharing ideas with us.

\section{Proof of Theorem 1.1}

Proof. First let us write, for any $n$ and $t$,

$$
\Pi_{n}(t)=\sum_{k=0}^{n-1}\left(\frac{t}{n}\right)^{k}\left(\sum_{0 \leq i_{1}<\ldots<i_{k} \leq n-1} A_{i_{1} \ldots A_{i_{k}}}\right)
$$

and notice that for any $k$,

$$
\left\|\left(\frac{t}{n}\right)^{k}\left(\sum_{0 \leq i_{1}<\ldots<i_{k} \leq n-1} A_{i_{1}} \ldots A_{i_{k}}\right)\right\| \leq\left(\frac{t}{n}\right)^{k} \sum_{0 \leq i_{1}<\ldots<i_{k} \leq n-1}\left\|A_{i_{1}}\right\| \ldots\left\|A_{i_{k}}\right\| .
$$

Notice that by commutativity

$$
\sum_{0 \leq i_{1}<\ldots<i_{k} \leq n-1}\left\|A_{i_{1}}\right\| \ldots\left\|A_{i_{k}}\right\|<\frac{1}{k !}\left(\sum_{k=0}^{n}\left\|A_{k}\right\|\right)^{k}
$$

so we have

$$
\left\|\left(\frac{t}{n}\right)^{k}\left(\sum_{0 \leq i_{1}<\ldots<i_{k} \leq n-1} A_{i_{1} \ldots A_{i_{k}}}\right)\right\| \leq \frac{\left|t^{k} \alpha^{k}\right|}{k !} .
$$

Hence, by the dominated convergence theorem, in order to prove the theorem, we only need to show that, for any $k$,

$$
\lim _{n \rightarrow+\infty} \frac{1}{n^{k}} \sum_{0 \leq i_{1}<\ldots<i_{k} \leq n-1} A_{i_{1}} \ldots A_{i_{k}}=\frac{1}{k !} A^{k} .
$$

We proceed by induction on $k$. The case $k=1$ is the hypothesis of the theorem. Let us assume that this property is true for a fixed integer $k$.

$$
\frac{1}{n^{k+1}} \sum_{0 \leq i_{1}<\ldots<i_{k}<l \leq n-1} A_{i_{1}} \ldots A_{i_{k}} A_{l}=\frac{1}{n^{k+1}} \sum_{l=k}^{n-1}\left(\sum_{0 \leq i_{1}<\ldots<i_{k}<\leq l-1} A_{i_{1}} \ldots A_{i_{k}}\right) A_{l}
$$


and by induction hypothesis there is a sequence $\left(\epsilon_{l}\right)_{l \in \mathbb{N}}$ going to zero such that for any $l$ :

$$
\left(\sum_{0 \leq i_{1}<\ldots<i_{k}<\leq l-1} A_{i_{1}} \ldots A_{i_{k}}\right)=\frac{l^{k}}{k !} A^{k}+l^{k} \epsilon_{l} .
$$

Hence

$$
\frac{1}{n^{k+1}} \sum_{0 \leq i_{1}<\ldots<i_{k}<l \leq n-1} A_{i_{1} \ldots} A_{i_{k}} A_{l}=\frac{1}{n^{k+1} k !} \sum_{l=k}^{n-1}\left(l^{k} A^{k}+l^{k} \epsilon_{l}\right) A_{l}
$$

and it follows that

$$
\frac{1}{n^{k+1}} \sum_{0 \leq i_{1}<\ldots<i_{k}<l \leq n-1} A_{i_{1}} \ldots A_{i_{k}} A_{l}=\frac{1}{n^{k+1} k !} A^{k} \sum_{l=k}^{n-1} l^{k} A_{l}+\frac{1}{n^{k+1} k !} \sum_{l=k}^{n-1} l^{k} \epsilon_{l} A_{l}
$$

So in order to get the result, we must prove that

$$
\lim _{n \rightarrow+\infty} \frac{1}{n^{k+1}} \sum_{l=k}^{n-1} l^{k} A_{l}=\frac{1}{k+1} A
$$

and

$$
\lim _{n \rightarrow+\infty} \frac{1}{n^{k+1}} \sum_{l=k}^{n-1} l^{k} \epsilon_{l} A_{l}=0 .
$$

Since the sequence $\left(\epsilon_{l}\right)_{n \in \mathbb{N}}$ goes to 0 as $l$ goes to $+\infty$, and since the mean of the norms of the $\left\|A_{i}\right\|$ are bounded by hypothesis, it is obvious that the first limit implies the second.

Let us write

$$
\frac{1}{n^{k+1}} \sum_{l=k}^{n-1} l^{k} A_{l}=\frac{1}{n} \sum_{l=k}^{n-1}\left(\frac{l}{n}\right)^{k} A_{l}
$$

and state the following.

Lemma 2.1. Let $\left(u_{n}\right)_{n \in \mathbb{N}}$ be a sequence with values in $\mathcal{M}_{d}(\mathbb{C})$ whose mean converges towards $L$ and let $g$ be a function in $\mathcal{C}^{1}(\mathbb{R})$. Then,

$$
\lim _{n \rightarrow+\infty} \frac{1}{n} \sum_{l=0}^{n-1} g\left(\frac{l}{n}\right) u_{l}=L \int_{0}^{1} g(t) d t .
$$

Applying this lemma to $\left(A_{l}\right)_{l \in \mathbb{N}}$ with $g: x \mapsto x^{k}$ yields the result.

Proof of Lemma 2.1. We start with an Abel transform. With the notations of the lemma, let us denote, for any integer $n$,

$$
S_{n}=\sum_{l=0}^{n} u_{l}
$$

and $S_{-1}=0$. For any $n$ in $\mathbb{N}$,

$$
\frac{1}{n} \sum_{l=0}^{n-1} g\left(\frac{l}{n}\right) u_{l}=\frac{1}{n} \sum_{l=0}^{n-1} g\left(\frac{l}{n}\right)\left(S_{l}-S_{l-1}\right)
$$


and so

$$
\frac{1}{n} \sum_{l=0}^{n-1} g\left(\frac{l}{n}\right) u_{l}=\frac{1}{n} \sum_{l=0}^{n-1} g\left(\frac{l}{n}\right) S_{l}-\frac{1}{n} \sum_{l=0}^{n-1} g\left(\frac{l}{n}\right) S_{l-1}
$$

which yields

$$
\frac{1}{n} \sum_{l=0}^{n-1} g\left(\frac{l}{n}\right) u_{l}=\frac{1}{n} \sum_{l=0}^{n-2} S_{l}\left(g\left(\frac{l}{n}\right)-g\left(\frac{l+1}{n}\right)\right)+\frac{1}{n} S_{n-1} \cdot g\left(\frac{n-1}{n}\right) .
$$

Now let us recall that $\lim _{n \rightarrow+\infty} \frac{1}{n} S_{n-1}=L$ hence there exists a sequence $\left(\epsilon_{l}\right)_{l \in \mathbb{N}}$ whose limit is zero such that for any $l$

$$
S_{l}=l\left(L+\epsilon_{l}\right) .
$$

This, in turn, yields

$$
\frac{1}{n} \sum_{l=0}^{n-1} g\left(\frac{l}{n}\right) u_{l}=\frac{1}{n} \sum_{l=0}^{n-2} l\left(L+\epsilon_{l}\right)\left(g\left(\frac{l}{n}\right)-g\left(\frac{l+1}{n}\right)\right)+\frac{1}{n}(n-1)\left(L+\epsilon_{n-1}\right) \cdot g\left(\frac{n-1}{n}\right) .
$$

Now let us remark that

$$
\lim _{n \rightarrow+\infty} \frac{1}{n}\left(n \epsilon_{n-1}-L-\epsilon_{n-1}\right) \cdot g\left(\frac{n-1}{n}\right)=0
$$

and that, since $g$ is differentiable, by the mean value theorem, for any couple of integers $l<n$, there exists a real $x$ in $\left[\frac{l}{n}, \frac{l+1}{n}\right]$ such that $g\left(\frac{l}{n}\right)-g\left(\frac{l+1}{n}\right)=\frac{g^{\prime}(x)}{n}$. The function $g^{\prime}$ being continuous, it is bounded on $[0,1]$, and since $\lim _{l \rightarrow+\infty} \epsilon_{l}=0$, we have

$$
\lim _{n \rightarrow+\infty} \frac{1}{n} \sum_{l=0}^{n-2} l \epsilon_{l}\left(g\left(\frac{l}{n}\right)-g\left(\frac{l+1}{n}\right)\right)=0 .
$$

So, in order to complete the proof of this lemma, we must understand the asymptotic behaviour of

$$
\frac{L}{n} \sum_{l=0}^{n-2} l\left(g\left(\frac{l}{n}\right)-g\left(\frac{l+1}{n}\right)\right)+L \cdot g\left(\frac{n-1}{n}\right) .
$$

First we write

$$
\frac{L}{n} \sum_{l=0}^{n-2} l\left(g\left(\frac{l}{n}\right)-g\left(\frac{l+1}{n}\right)\right)+L \cdot g\left(\frac{n-1}{n}\right)=\frac{L}{n} \sum_{l=1}^{n-2} g\left(\frac{l}{n}\right)-\frac{(n-2)}{n} L \cdot g\left(\frac{n-1}{n}\right)+L \cdot g\left(\frac{n-1}{n}\right) \text {. }
$$

From here on, noticing that $\frac{1}{n} \sum_{l=1}^{n-2} g\left(\frac{l}{n}\right)$ is a Riemann sum yields the result, we indeed have

$$
\lim _{n \rightarrow+\infty} \frac{1}{n} \sum_{l=0}^{n-1} g\left(\frac{l}{n}\right) u_{l}=L \int_{0}^{1} g(t) d t
$$




\section{Applications to dynamics}

Corollary 3.1. Let $(X, \mathcal{B}, \mu, T)$ be a measured dynamical system, $\mu$ being an ergodic $T$-invariant probability measure. Let $A$ be function from $X$ to $\mathcal{M}_{d}(\mathbb{C})$ such that each $A_{i, j}$ is in $L^{1}(X, \mu)$. Then, for almost every $x$ in $X$,

$$
\lim _{n \rightarrow+\infty}\left(I_{d}+\frac{t}{n} A(x)\right) \ldots\left(I_{d}+\frac{t}{n} A \circ T^{n-1}(x)\right)=\exp \left(t \int_{X} A(x) d \mu(x)\right) .
$$

Proof. This is just a matter of writing Theorem 1.1 using Birkhoff's pointwise ergodic theorem.

The fact that this theorem applies to generic points of ergodic probability measures is useful to understand some dynamical systems as we illustrate with the following corollary which gives the asymptotic law of a "random walk" on horocycles of the hyperbolic disc.

Corollary 3.2. Let $A_{1}=\left(\begin{array}{ll}0 & 1 \\ 0 & 0\end{array}\right)$ and $A_{2}=\left(\begin{array}{ll}0 & 0 \\ 1 & 0\end{array}\right)$. Let $\mu$ be a shift-invariant ergodic probability measure on $\{1,2\}^{\mathbb{N}}$. Then, for $\mu$ almost every $x$ in $\{1,2\}^{\mathbb{N}}$, and every $t$ in $\mathbb{R}$,

$$
\left(I_{2}+\frac{t}{n} A_{x_{0}}\right) \ldots\left(I_{2}+\frac{t}{n} A_{x_{n-1}}\right)=\left(\begin{array}{cc}
\cosh \left(\frac{t}{\sqrt{\mu([1]) \mu([2])}}\right) & \sqrt{\frac{\mu([2])}{\mu([1])}} \sinh \left(\frac{t}{\sqrt{\mu([1]) \mu([2])}}\right) \\
\sqrt{\frac{\mu([0])}{\mu([2])}} \sinh \left(\frac{t}{\sqrt{\mu([1]) \mu([2])}}\right) & \cosh \left(\frac{t}{\sqrt{\mu([1]) \mu([2])}}\right)
\end{array}\right)
$$

Proof. One easily computes $\exp \left(\begin{array}{cc}0 & t \mu([1]) \\ t \mu([2]) & 0\end{array}\right)$ to get the desired result.

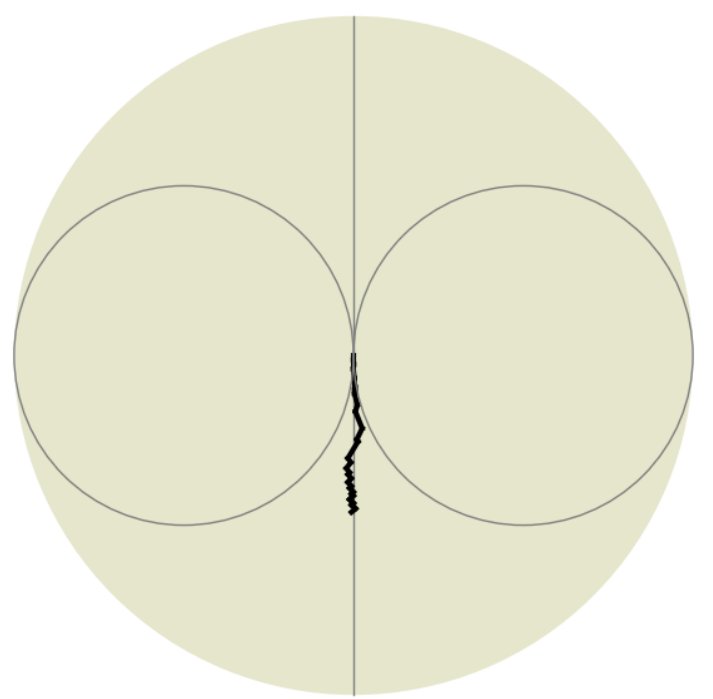

Figure 1: An illustration of a random hyperbolic walk 
Remark 3.3. Remark that Corollary 3.2 can be interpreted in a geometric way given that $I_{2}+A_{1}$ and $I_{2}+A_{2}$ are generators of $S L_{2}(\mathbb{Z})$. Notice that taking, for instance, $\mu$ to be the symmetric Bernoulli measure on $\{1,2\}^{\mathbb{N}}$, one gets $\Pi_{X}(t)=\left(\begin{array}{ll}\cosh \left(\frac{t}{2}\right) & \sinh \left(\frac{t}{2}\right) \\ \sinh \left(\frac{t}{2}\right) & \cosh \left(\frac{t}{2}\right)\end{array}\right)$ which is conjugated to $\left(\begin{array}{cc}e^{\frac{t}{2}} & 0 \\ 0 & e^{-\frac{t}{2}}\end{array}\right)$ by a rotation of angle $\pi / 4$. Hence almost every "random walk" (with simultaneous renormalization) on two horocycles of the hyperbolic disc converges towards a unique point on the geodesic represented by the vertical diameter of the disc as illustrated on Figure $1^{1}$.

\section{References}

[1] Yves Benoist and Jean-François Quint. Random walks on reductive groups, volume 62 of Ergebnisse der Mathematik und ihrer Grenzgebiete. 3. Folge. A Series of Modern Surveys in Mathematics [Results in Mathematics and Related Areas. 3rd Series. A Series of Modern Surveys in Mathematics]. Springer, Cham, 2016.

[2] J. Emme and P. Hubert. Central limit theorem for probability measures defined by sum-ofdigits function in base 2. Preprint, (arXiv:1605.06297), 2016.

[3] Alex Furman. Random walks on groups and random transformations. In Handbook of dynamical systems, Vol. 1A, pages 931-1014. North-Holland, Amsterdam, 2002.

[4] François Ledrappier. Some asymptotic properties of random walks on free groups. In Topics in probability and Lie groups: boundary theory, volume 28 of CRM Proc. Lecture Notes, pages 117-152. Amer. Math. Soc., Providence, RI, 2001.

\footnotetext{
${ }^{1}$ The calculations are made in the hyperbolic plane but the picture is presented in the disc since it is more symmetric.
} 